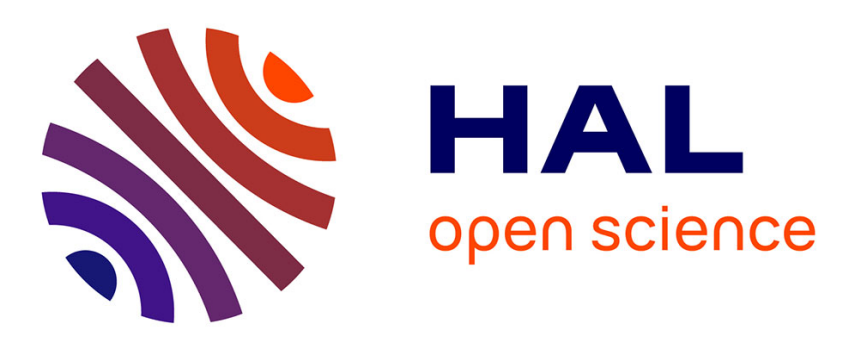

\title{
Sound simulation-based design optimization of brass wind instruments
}

Robin Tournemenne, Jean-François Petiot, Bastien Talgorn, Joel Gilbert, Michael Kokkolaras

\section{- To cite this version:}

Robin Tournemenne, Jean-François Petiot, Bastien Talgorn, Joel Gilbert, Michael Kokkolaras. Sound simulation-based design optimization of brass wind instruments. Journal of the Acoustical Society of America, 2019, 145 (6), pp.3795-3804. 10.1121/1.5111346 . hal-01963668

\section{HAL Id: hal-01963668 \\ https://hal.inria.fr/hal-01963668}

Submitted on 30 Sep 2019

HAL is a multi-disciplinary open access archive for the deposit and dissemination of scientific research documents, whether they are published or not. The documents may come from teaching and research institutions in France or abroad, or from public or private research centers.
L'archive ouverte pluridisciplinaire HAL, est destinée au dépôt et à la diffusion de documents scientifiques de niveau recherche, publiés ou non, émanant des établissements d'enseignement et de recherche français ou étrangers, des laboratoires publics ou privés. 


\section{Sound simulation-based design optimization of brass wind instruments}

Robin Tournemenne, ${ }^{1}$ Jean-François Petiot, ${ }^{2}$ Bastien Talgorn, ${ }^{3}$ Joël Gilbert, ${ }^{4}$ and Michael Kokkolaras ${ }^{3}$

${ }^{1)}$ Magique 3D Team, Inria Bordeaux Sud Ouest, 200 avenue de la vieille tour, 33405 Talence Cedex, France ${ }^{\mathrm{a}}$

${ }^{2}$ École Centrale de Nantes, LS2N, UMR CNRS 6004, 1 rue de la Noë, 44321 Nantes Cedex 3, France ${ }^{\mathrm{b}}$

${ }^{3}$ Department of Mechanical Engineering, McGill University, Montréal, QC H3A OC3, Canada $^{\mathrm{c}}$

${ }^{4}$ Laboratoire d'Acoustique de l'Université du Mans, UMR CNRS 6613, avenue Olivier Messiaen, 72085 Le Mans Cedex 09, France ${ }^{\mathrm{d}}$ 
We present a method for optimizing the inner shape of brass instruments using sound simulations. This study considers different objective functions and constraints (representative of both the intonation and the spectrum of the instrument) for a relatively large number of design variables. A complete physics-based model, taking into account the instrument and the musician embouchure, is used to simulate permanent regimes of sounds by means of the harmonic balance technique, the instrument being represented by its input impedance. The design optimization variables are related to the geometrical dimensions of the resonator. The embouchure's parameters are varied during the optimization procedure to obtain an average behavior of the instrument. The objective and constraint functions of the optimization problem are evaluated using the physics-based simulation model, which is computationally expensive. Moreover, the gradients of the objective and constraint functions can be discontinuous, unavailable, or hard to approximate reliably. Therefore, we employ a surrogate-assisted derivative-free optimization strategy using the mesh adaptive direct search algorithm (MADS). One example of a Bb trumpet's bore is used to demonstrate the effectiveness of the design optimization approach: the obtained results improve previously reported objective function values significantly.

\footnotetext{
a) robin.tournemenne@inria.fr;

b) jean-francois.petiot@1s2n.fr;

c) michael.kokkolaras@mcgill.ca;

d)joel.gilbert@univ-lemans.fr;
} 


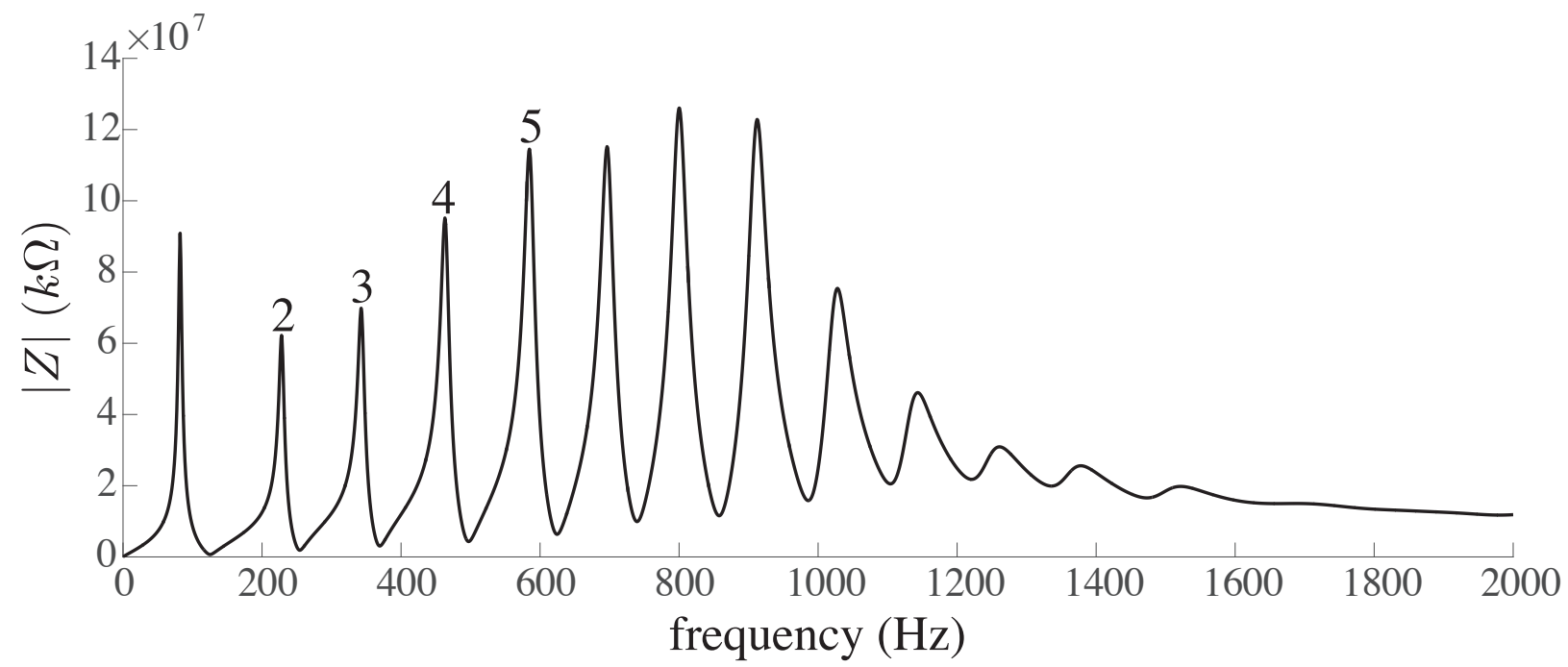
instrument manufacturers. Many prototypes are required to ensure that quality attributes such as intonation, ease of emission, timbre, projection, etc. are adequate at each stage of the development process. Numerical acoustics models may be helpful in shortening development cycles by minimizing the manufacturing of costly prototypes. For example, in the case of brass instruments, several studies propose the use of numerical modeling to predict quality and guide the design process (Campbell, 2004; Macaluso and Dalmont, 2011).

The dominant physical quantity impacting the sound quality of a brass instrument is its input impedance (cf. Figure 1). Input impedance is the frequency-dependent quotient of pressure and

FIG. 1. Simulated input impedance $Z$ of a $\mathrm{B} b$ trumpet (magnitude), highlighting resonances 2, 3, 4, and 5 of the instrument

\section{INTRODUCTION}

The development of innovative and higher-quality designs is crucial to the viability of musical

volume flow at the instrument entry plane, and, at first approximation, is the result of the in- 
strument's interior shape (the bore). Many works have focused on modeling input impedance, e.g., (Caussé et al., 1984).

The impact of input impedance on sound quality is acknowledged in (Campbell, 2004), where, in a first approximation attempt, playing frequencies are governed mainly by corresponding impedance peaks (Eveno et al., 2014). Beyond influencing the instrument intonation, impedance also determines an instrument's timbre and playability, notably due to the height and bandwidth of peaks.

With this in view, several researchers have used input impedance to design an instrument's inner shape using an optimization approach. Following Kausel's successful reconstruction of a trumpet bore using the Rosenbrock algorithm (Kausel, 2001), Noreland optimized the instrument's intonation with a hybrid scheme for the input impedance model and shape constraints (Noreland et al., 2010). Braden also optimized the intonation and the input impedance peak heights of a trombone using a multi-modal input impedance model (Braden et al., 2009), while Macaluso optimized and built a near-perfect harmonic trumpet (Macaluso and Dalmont, 2011). Some studies investigated the relationship between input impedance features and psycho-acoustic criteria: Poirson optimized the trumpet using objective functions based on the input impedance and targets defined by trumpet players preferences (Poirson et al., 2007). Guillauteau looked for empirical relations between playing frequencies and resonance frequencies to optimize clarinets (Guilloteau, 2015).

However valuable, these works focused exclusively on instrument performance, neglecting a crucial element in sound production: the musician embouchure. In particular, the studies of Eveno et al. showed that the relationship between the resonance frequencies of the impedance and the actual frequencies of the sounds played by musicians can vary significantly (Eveno et al., 2014). 
Although the impedance of an instrument provides interesting information about sound quality, prediction of "playability" and sound qualities of brasses based solely on impedance remains difficult.

A second approach is based on a holistic model of the physical phenomenon, coupling the instrument and the musician embouchure, to produce sound simulations representative of the instrument quality. Using this approach, the authors integrate sound simulations in the optimization process. These simulations are obtained from a physics-based model to account for the interaction of the instrument with a virtual musician embouchure (Tournemenne et al., 2017). In a previous study, the instrument's intonation was optimized based on simulated playing frequencies (Tournemenne et al., 2017). Two examples were considered, optimizing 2 and 5 of the bore's geometrical parameters, respectively; results were quite encouraging.

The main objective of the present paper is to extend this new optimization paradigm in order to assess both its potential and limitation. The two main novelties of this paper are the optimization of criteria based on the instrument sounds spectra, and the inclusion of constraints in the problem formulation. Three other contributions are noticeable: i) an improved version of the optimization method has been implemented, ii) a new solver is introduced, and iii) the performance limits of the optimization method are tested by considering 10 geometrical variables of the bore. A trumpet is used as a representative brass instrument to demonstrate the proposed design optimization method.

The paper is organized as follows. We first present extensive details on the physics-based model and the simulation technique. We then formulate the optimization problems and describe the principles of the MADS algorithm and the framework for surrogate-assisted optimization. 


\begin{abstract}
Finally, we conduct a case study concerning the shape optimization of a trumpet with ten design variables and draw conclusions.
\end{abstract}

\title{
II. TRUMPET MODELING
}

In this study, we utilize an elementary model of a brass instrument under playing conditions: the vibrating lips are modeled as a one-degree-of-freedom (1-DOF) outward-striking valve, nonlinearly coupled to the air column of the brass instrument. This elementary model is a good compromise between simplicity and efficiency. While the 1-DOF model cannot model real musician lips exactly, (Yoshikawa, 1995) it is able to mimic a large range of playing phenomena (see for example the pioneering work of Elliot and Bowsher (1982), (Elliott and Bowsher, 1982) or the more recent works of Petiot et al. (2013) (Petiot and Gilbert, 2013) and Velut et al. (2017) (Velut et al., 2017)). Similar to Chen and Weinreich (1996), (Chen and Weinreich, 1996) we argue that while the lips may not be entirely modeled by a 1-DOF model, most characteristic behaviors of brasses can be reproduced by an outward striking reed.

Our physics-based model of the trumpet is based on Equations (1), (2), and (3), which all depend on three periodic variables: the opening height $h(t)$ of the two lips, the volume flow $u(t)$ of the air jet through the lip channel and the pressure $p(t)$ in the mouthpiece (cf. Figure 2).

$$
\hat{p}(j \omega)=Z(j \omega) \hat{u}(j \omega)
$$

$$
\frac{d^{2} h(t)}{d t^{2}}+\frac{2 \pi f_{\ell}}{Q_{\ell}} \frac{d h(t)}{d t}+\left(2 \pi f_{\ell}\right)^{2}\left(h(t)-h_{0}\right)=\frac{P_{m}-p(t)}{\mu_{\ell}}
$$




$$
u(t)=b h^{+}(t) \operatorname{sign}\left(P_{m}-p(t)\right) \sqrt{\frac{2\left|P_{m}-p(t)\right|}{\rho}}
$$

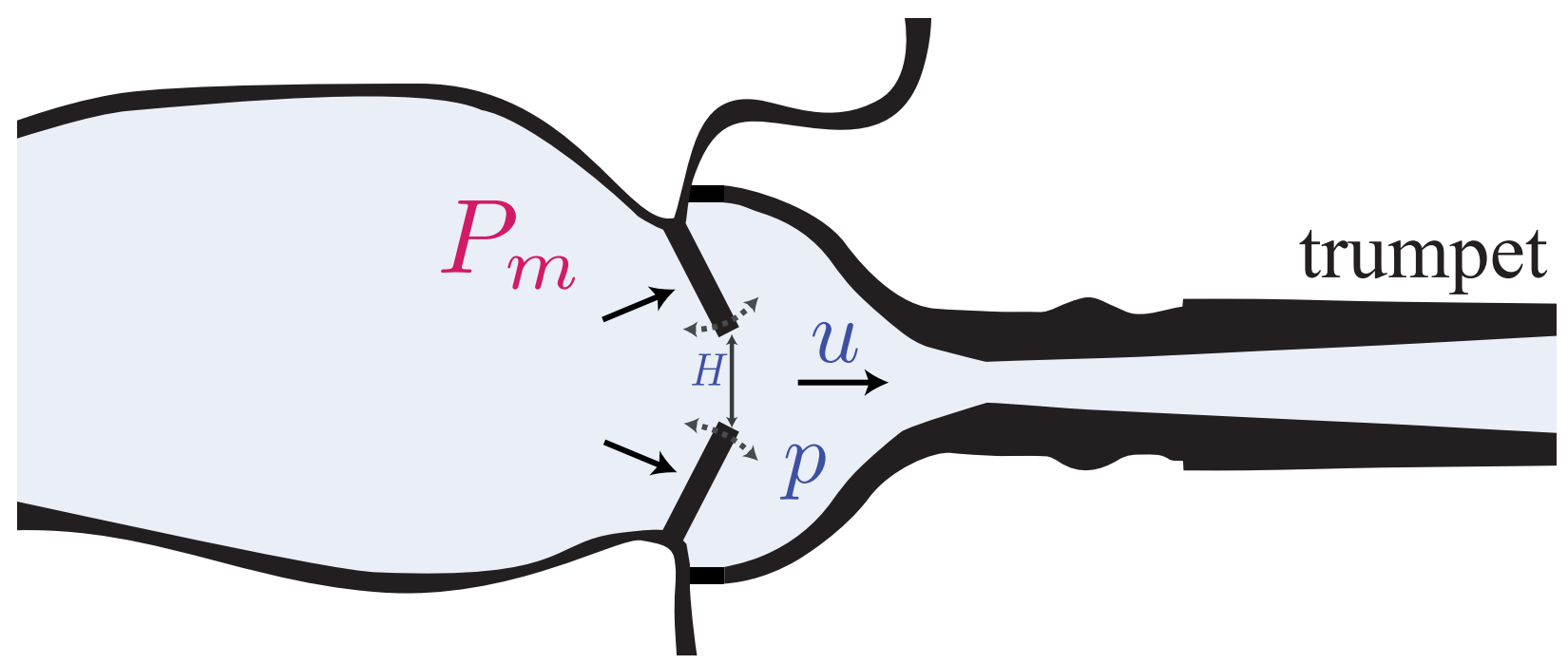

FIG. 2. Representation of the outward striking model of the lips, with the definition of the variables of the physical model: $P_{m}$ (pressure in the mouth), $h$ (lip aperture), $u$ (volume flow), $p$ (pressure in the mouthpiece) (color online)

These three equations express the acoustic impedance of the resonator, a simple harmonic oscillator for the lips model, and the coupling between the lips and the trumpet, respectively. Equation (3) shows two non-linearities: the square root originating from the Bernoulli equation, and the positive part of the lip aperture $h^{+}=\max (h, 0)$ modelling the closed lips. Several parameters are included in this model: air density $\rho$, input impedance $Z$ of the trumpet, the parameters concerning musician embouchure which are $P_{m}$ (the static overpressure in the mouth), $f_{\ell}$ (the resonance frequency of the lips), $\mu_{\ell}$ (the area density of the lips), $b$ (the width of the lips), $h_{0}$ (the rest value of the opening height of the lips) and $Q_{\ell}$ (the quality factor of the resonance of the lips). Input 
impedance $\mathrm{Z}$ is computed using the transfer matrix method considering plane wave propagation, visco-thermal losses (Caussé et al., 1984), and a radiation function under an infinite plane baffle hypothesis.

It is important to assess the validity of this 1-DOF model by indicating which behaviors of brasses can be reproduced adequately with simulations and which ones cannot. Previous results using this elementary model (Petiot and Gilbert, 2013) showed that the trumpet sounds simulated are dissimilar to the real trumpet sounds played by a musician. In particular, sound spectra in permanent regime are very different. This can be explained by inherent limitations of the model. A first limitation concerns the linear approximation of sound propagation in the brass resonator defined by its acoustic impedance (Equation (1)). When the instrument is played loudly and brassy, this approximation is no longer valid, and the nonlinear propagation needs to be taken into account. (Myers et al., 2012)

A second limitation concerns the lip model, which simplifies the complicated real-lips motion (Bromage et al., 2010; Martin, 1942; Yoshikawa, 1995) with a 1-DOF (Equation (2)). Two-degrees-of-freedom (2-DOF) models have been considered for time domain simulation (Adachi and Sato, 1996; Boutin et al., 2015). Furthermore, measured mechanical responses of artificial (Cullen et al., 2000) and real brass-players lips (Newton et al., 2008) revealed that a pair of mechanical resonances requires a 2-DOF model in order to be consistent with near threshold oscillations (Cullen et al., 2000). However, although additional terms can theoretically be added to the 1-DOF model, the difficulty lies in selecting realistic values for the additional parameters (Velut et al., 2017).

A third limitation relates to the assumption that the volume flow u (Equation (3)), which controls the valve effect, is proportional to the opening height $h$ between the lips. Experimental data 
reported in (Bromage et al., 2010) for a large set of playing frequencies and sound levels showed that the relationship may be exponential instead of linear, and dependent on the pitch and dynamic level of the note played.

Nevertheless, even if nonrealistic for the spectrum of trumpet sounds, previous studies confirm that this elementary model behaves in agreement with the main physical principles that govern the playing of brasses (Petiot and Gilbert, 2013; Poirson et al., 2005). In particular, results presented in (Petiot and Gilbert, 2013) showed that the elementary model is able to produce differences between instruments according to playing frequency, spectral centroid, and evolution of the spectral centroid with the playing dynamics that are, on average, in agreement with the differences noticed when a real trumpet is playing. This results justify the use of this elementary model in an optimization process for objective functions based on intonation, spectral centroid, or the evolution of the spectral centroid.

Numerical solutions of this system of equations are obtained using the harmonic balance technique to simulate the sound created by a given trumpet (defined by its input impedance $Z$ ) for a given "virtual musician embouchure" (defined by its control parameters). The harmonic balance technique considers the sound's permanent regime (steady state); since the latter is periodic, the truncated pressure is given by

$$
p(t)=A_{0}+\sum_{n=1}^{N} A_{n} e^{i 2 \pi n F t}+A_{n}^{*} e^{-i 2 \pi n F t} .
$$

The unknowns, i.e., the amplitudes of the harmonics $A_{n}$ and the playing frequency $F$, are determined using Newton's method (Gilbert et al., 1989).

To perform a sound simulation, it is necessary to define relevant values (i.e., values that lead to a convergence towards a steady-state sound for a given note) for the parameters of the musician 
embouchure. For a given note, experience shows that countless embouchures may lead to a steadystate note. The choice of the range of the parameters is based both on numerical tests of the simulations and on measurements of real trumpet players. The three variables $P_{m}, \mu_{\ell}$, and $f_{\ell}$ are considered as control parameters of the simulations, and constitute the virtual embouchure. The pressure $P_{m}$ in the mouth influences mainly the dynamics of a simulated sound and ranges from 1 to $12 \mathrm{kPa}$ (Fletcher and Tarnopolsky, 1999). In the following numerical experiments, we partitioned this range into three parts running from 1 to $5 \mathrm{kPa}$ for what we call piano $(p)$ dynamics, 5 to $9 \mathrm{kPa}$ for mezzoforte $(m f)$ dynamics and 9 to $12 \mathrm{kPa}$ for fortissimo $(f f)$ dynamics. The frequency of the lips $f_{\ell}$ enables the selection of the played regime (note): the higher the value of $f_{\ell}$, the higher the simulated regime. Exploration tests led to a range for $f_{\ell}$ that spans from $130 \mathrm{~Hz}$ to $480 \mathrm{~Hz}$ to simulate the $2^{\text {nd }}, 3^{\text {rd }}, 4^{\text {th }}$ and $5^{\text {th }}$ regime of the $\mathrm{B} b$ trumpet with no valve pressed, the regimes considered in this study. These regimes correspond to the musical notes $\mathrm{B} b 3, \mathrm{~F} 4, \mathrm{~B} b 4$, D5-concert-pitch. Finally, in order to produce many different sounds for every regime, we add variability to the embouchure making $\mu_{\ell}$ a control parameter of the simulations, ranging from 1 to $6 \mathrm{~kg} / \mathrm{m}^{2}$ (Cullen et al., 2000). In our study, the values of $b, Q_{\ell}$, and $h_{0}$ are the same for every simulation (Cullen et al., 2000).

The values of the control parameters considered in this study are summarized in Table I.

Given that above $3000 \mathrm{~Hz}$ the impedance magnitude is flat (see Figure 1), it is not relevant to consider many harmonics for the sound simulation. The highest studied note being D5 (587 $\mathrm{Hz}$ ), we chose to simulate our permanent regime with only $N=6$ harmonics. In conclusion, for a given trumpet (characterized by its input impedance $Z$ ) and for a virtual musician embouchure (characterized by the parameters $P_{m}, \mu_{\ell}, f_{\ell}, b, h_{0}$, and $Q_{\ell}$ ), the simulation may generate one note (if 
TABLE I. Values of the control parameters for the simulations considered in the study (virtual musician embouchure)

\begin{tabular}{|c|c|c|}
\hline Parameter & Symbol (units) & Value \\
\hline Resonance frequency of the lips & $f_{\ell}(\mathrm{Hz})$ & 130 to 480 \\
\hline Mass per area of the lips & $\mu_{\ell}\left(k g / m^{2}\right)$ & 1 to 6 \\
\hline Pressure in the mouth & $P_{m}(\mathrm{kPa})$ & 1 to 12 \\
\hline Width of the lips & $b(\mathrm{~mm})$ & 10 \\
\hline Rest value of the opening height & $h_{0}(\mathrm{~mm})$ & 0.1 \\
\hline Quality factor of the resonance & $Q_{\ell}$ & 3 \\
\hline
\end{tabular}

It is important to mention that the computed sound $p(t)$ corresponds to the sound in the mouthpiece. According to Benade (Benade, 1966), a relevant spectrum transformation function could be defined to compute the sound outside the instrument. The difficulty lies in the definition of the radiated pressure, relevant from a perceptual point of view. For the optimization considered in this work, the well-defined pressure in the mouthpiece is deemed sufficient. It is also important to mention that the convergence of the simulation toward auto-oscillations is not ensured for a given shape of the resonator and embouchure. The search of convenient embouchures is a complex task, described in the following section. 


\section{OPTIMIZATION PROBLEM FORMULATION}

The design optimization problem of an instrument can be formulated as the search for the optimal geometry minimizing an objective function:

$$
\min _{\mathbf{x} \in \Omega} J(\mathbf{x})
$$

where $J: \mathbb{R}^{n} \rightarrow \mathbb{R}$ is the objective function, and the vector $\mathbf{x} \in \mathbb{R}^{n}$ includes the design optimization variables. The design space $\Omega$ is a subset of $\mathbb{R}^{n}$ delimited by box (bound) constraints. The design optimization variables are the geometric parameters that define the inner shape of the bore. To facilitate the input impedance calculations, the bore is approximated by a series of conical and cylindrical waveguide segments. Consequently, $\mathbf{x}$ is a vector of geometric quantities such as the lengths and radii of cylinders or cones. The design space $\Omega$ may be modified to obtain viable trumpet shapes.

Two classes of objective functions are available considering our physics-based model and the harmonic balance technique: descriptors based on playing frequencies and descriptors based on sounds spectra. Many quantities based on frequency and sound spectrum can be found in the literature; however, there is no consensus in the community regarding their influence on the instrument's musical quality. These disagreements notwithstanding, this paper considers three different objective functions based on intonation and spectral centroid given their recognized impact on the instrument quality (Deutsch, 2013). 


\section{A. Objective functions}

Figure 3 describes the flowchart of the process for optimizing the shape of a trumpet bore using physics-based sound simulations. Input impedance is computed for a design vector $\mathbf{x}$ representing Set $\varphi$ of embouchures

$$
\left(P_{m}, \mu_{\ell}, f_{\ell}\right)
$$

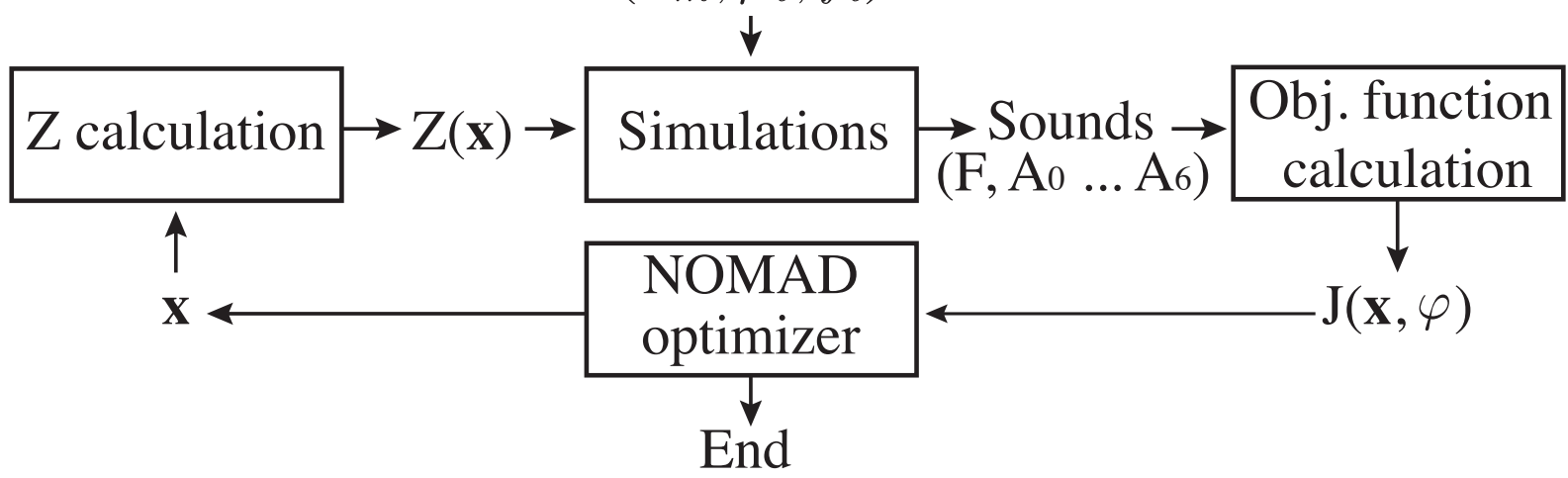

FIG. 3. Flowchart of the optimization process

the resonator's geometry. This study focuses on the average behavior of the instrument across a panel of embouchures for each note (Bb3, F4, Bb4, D5). Another approach based on ideal embouchures for each note could have been adopted, which would be an interesting future line of research. Consequently, for the $P$ notes ( $P=4$ in this study), the harmonic balance technique simulates many sounds based on the calculated input impedance $Z(\mathbf{x})$ and a set of virtual embouchures $\varphi_{i}$ for each note $i$. The set

$$
\varphi=\bigcup_{i=1}^{P} \varphi_{i}
$$

represents the entire set of embouchures used to evaluate one instrument (see section III C for details). An objective function $J$ is then computed using the playing frequencies $F$ or the harmonics amplitudes $A_{n}$ produced by the $\operatorname{card}(\varphi)$ simulations ( $\operatorname{card}(\cdot)$ denotes cardinality of a set, i.e., the 
number of elements in a set). The value of the objective function (and the values of the constraint functions introduced later) are provided to the optimization algorithm (MADS, implemented in the NOMAD software package (Le Digabel, 2011)), which will propose a new design vector $\mathbf{x}$ (see Section IV for details). The new design candidate $\mathbf{x}$ is evaluated in the same manner, and the process is iterated until the optimization algorithm termination criterion is satisfied.

\section{Objective function based on intonation}

For each note $i$, the average (across different embouchures) playing frequency $\overline{F_{i}}(\mathbf{x}, \varphi)$ at mezzoforte dynamics is computed. The intonation of the note is assessed by the deviation of the actual playing frequency, as simulated using the physics-based model, from the expected playing frequency. This objective function relies on a reference note from which the ideal and actual musical distance is computed. We chose for reference the $4^{\text {th }}$ regime of the trumpet with no valve pressed (B b4, concert pitch), given that it is the usual tuning note of the instrument. The equal-tempered scale is used to define the ideal distance between the studied note $i$ and the reference note (Tournemenne et al., 2017).

For every note $i$, we compute the equal-tempered deviation (ETD) between the average frequency of the $\mathrm{i}^{\text {th }}$ note $\bar{F}\left(\mathbf{x}, \varphi_{\mathbf{i}}\right)$ and the reference frequency $\bar{F}\left(\mathbf{x}, \varphi_{\mathbf{r}}\right)$ as

$$
\operatorname{ETD}_{i}(\mathbf{x}, \varphi)=\alpha_{r \rightarrow i}-1200 \log _{2}\left(\frac{\bar{F}\left(\mathbf{x}, \varphi_{\mathbf{i}}\right)}{\bar{F}\left(\mathbf{x}, \varphi_{\mathbf{r}}\right)}\right)
$$

where $\alpha_{r \rightarrow i}$ is the ideal difference between the reference note $r$ and the targeted note $i$ given by the equal-tempered scale (-500 cents for example between Bb4 and F4). The objective function $J_{1}(\mathbf{x}, \varphi)$ for the whole instrument is the average of the absolute deviation across the $(P-1)$ notes 
(notice that the deviation between the reference note and the $4^{\text {th }}$ note is always equal to zero, given that it is the tuning note)

$$
J_{1}(\mathbf{x}, \varphi)=\frac{1}{P-1} \sum_{i \in \text { Notes }}\left|\operatorname{ETD}_{i}(\mathbf{x}, \varphi)\right|
$$

\section{Objective function based on the spectral centroid}

The average spectral centroid $\overline{\operatorname{SC}}(\mathbf{x}, \varphi)$ at mezzoforte dynamics for every note is computed according to the 6 harmonic amplitudes $A_{n}$ of each simulated sound

$$
\overline{\mathrm{SC}}(\mathbf{x}, \varphi)=\frac{1}{\operatorname{card}(\varphi)} \sum_{E_{v} \in \varphi} \operatorname{SC}\left(\mathbf{x}, E_{v}\left(P_{m}, \mu_{l}, f_{l}\right)\right),
$$

where $E_{v}$ is an embouchure of the virtual musician with

$$
\operatorname{SC}\left(\mathbf{x}, E_{v}\right)=\frac{\sum_{n=1}^{6}\left|n A_{n}\left(\mathbf{x}, E_{v}\right)\right|}{\sum_{n=1}^{6}\left|A_{n}\left(\mathbf{x}, E_{v}\right)\right|}
$$

Consequently, $\mathrm{SC}\left(\mathbf{x}, E_{v}\right)$ spans from 1 to 6 , representing the normalized spectral centroid. In this work, we decided to look for the instruments having the highest spectral centroid. These instruments would generally be considered as bright by musicians (Poirson et al., 2005). This is a somewhat arbitrary choice and other relevant descriptor/target may be found, although such consideration is out of the scope of the paper.

Consequently, the objective function $J_{2}(\mathbf{x}, \varphi)$ is

$$
J_{2}(\mathbf{x}, \varphi)=\overline{\mathrm{SC}}(\mathbf{x}, \varphi)
$$

\section{Objective function based on the spectral centroid dynamics}

This descriptor represents the ability of the instrument to maximise the spectral centroid difference between a piano $(p)$ and a fortissimo $(f f)$ dynamics. The idea is to find the instrument 
producing bright notes for high dynamics (high SC) while keeping a dark sound (low SC) for low dynamics. The average spectral centroid for each note and each piano $\left(\overline{\operatorname{SC}_{p}}\left(\mathbf{x}, \varphi_{i}\right)\right)$ and fortissimo $\left(\overline{\operatorname{SC}_{f f}}\left(\mathbf{x}, \varphi_{i}\right)\right)$ dynamics are computed. The objective function is then formulated as

$$
J_{3}(\mathbf{x}, \varphi)=\sum_{i \in \text { Notes }} \frac{\overline{\operatorname{SC}_{f f}}\left(\mathbf{x}, \varphi_{i}\right)-\overline{\mathrm{SC}_{p}}\left(\mathbf{x}, \varphi_{i}\right)}{P}=\sum_{i \in \text { Notes }} \frac{\Delta \overline{\mathrm{SC}}_{i}}{P}
$$

For this descriptor, the note D5 concert-pitch has been discarded because of the difficulty in simulating it for low dynamics. A more application-oriented study considering the entire instrument's tessitura should account for these kind of difficulties.

\section{B. Optimization problems}

In summary, we consider three different optimization problems labelled Int, SC Int, and SC Dyn. They represent instrument design problems that are realistic from a musician point of view:

- Int: intonation improvement:

$$
\min _{\mathbf{x} \in \Omega} J_{1}(\mathbf{x})
$$

- SC Int: spectral centroid improvement under intonation constraint:

$$
\max _{\mathbf{x} \in \Omega} J_{2}(\mathbf{x}) \text { subject to } J_{1}(\mathbf{x}) \leq J_{1_{\max }}
$$

- SC Dyn: spectral centroid dynamics improvement:

$$
\max _{\mathbf{x} \in \Omega} J_{3}(\mathbf{x})
$$

The constraint function of the SC Int problem is the objective function of the Int problem. It represents the scenario where musicians are willing to trade some of their instrument's intonation quality for a more brighter timbre. 


\section{Finding playable embouchures}

The main challenge lies in the simulation of many different sounds (represented by their permanent regimes) during the numerical evaluation of the objective function. Practically, for each geometry $\mathbf{x}$, we need to find suitable virtual embouchures leading to convergence toward permanent regime. Furthermore, similar to an inexp heed player that would blow even the most well-designed trumpet with a terrible sound, the virtual embouchure must be carefully selected in order to produce realistic sounds. No analytical approach exists to deal with this challenge and simple solutions always fall short. For example, it is not possible to define, a priori, a fixed list of virtual embouchures that will be used for every geometry $\mathbf{x}$, because experience shows that the intersection of the sets of virtual embouchures leading to convergence toward a permanent regime for each geometry may be empty. It is far too expensive to process a complete fine grid of the 3 virtual embouchure parameters for every geometry $\mathbf{x}$. Consequently, a rigorous preprocessing of the simulations is undertaken to help the simulations obtain a set of appropriate embouchures that converge toward adequate sounds for every geometry $\mathbf{x}$.

This preprocessing is based on an exploration of the area of the design space $\Omega$ augmented by the 3 embouchure parameters leading to convergence of the sound simulation. If $\mathbf{x}$ is in $\mathbb{R}^{2}$, the space to explore has 5 dimensions: 2 geometric variables and 3 embouchure variables $\left(P_{m}, \mu_{l}\right.$, $f_{l}$ ). To explore this space, a five-dimensional Latin hypercube is built and the harmonic balance technique tries to simulate every sample.

In order to discard the simulations of unrealistic sounds mentioned above, we use a criterion representing the amplitude of the simulated sound relatively to the pressure in the mouth. If the 
amplitudes of the harmonics are large enough relative to the mouth pressure $P_{m}$ produced by the virtual musician, the sound is considered appropriate

$$
\frac{\sqrt{\sum_{n=1}^{6} A_{n}^{2}}}{P_{m}} \geq \alpha .
$$

Given the exploration and the criterion, an empirical method finds adequate virtual embouchures for any geometry $\mathbf{x}$ of the design space. There are two key differences between the preprocessing presented here and that reported in (Tournemenne et al., 2017): i) the definition of the criterion threshold $\alpha$ based on live recordings and ii) a more robust technique used to define the set of virtual embouchures $\varphi$ for any bore, both summed up in the following paragraph.

The live recordings of 3 helped trumpeters playing several times the 4 notes allowed us to estimate the standard deviation of playing frequency for each note. We then defined $\alpha$ in order to obtain simulations having approximately the same standard deviation of playing frequency. In practice we defined one $\alpha$ per note and dynamic ( $p, m f, f f)$; its value ranges from 0.85 (D5) to $1.17(\mathrm{Bb} 3)$. During the optimization, the procedure defining $\varphi$ for every bore relies on a maximal distance from the corresponding cloud of successful embouchures found during the preprocessing, above which a virtual embouchure is discarded. In the interest of keeping the length of this paper reasonable, we point the interested reader to the manual accompanying the source code repository for a detailed description of the procedure (framagit.org/rtournem/Brass0ptimUsingSounds).

It is important to mention that for numerical reasons, the quantities $\bar{F}\left(\mathbf{x}, \varphi_{\mathbf{i}}\right)$ and $\overline{\mathrm{SC}}(\mathbf{x}, \varphi)$ are average values across a finite set of embouchures $\varphi$, randomly chosen and selected by the preprocessing. The consequence is that the objective function $J(\mathbf{x})$ is non-deterministic, i.e., different objective function values may be obtained for the same $\mathbf{x}$. In practice, a set of 1000 embouchures are simulated per note per dynamic range in order to keep the standard deviation on the numerical 
estimation of $J(\mathbf{x})$ as low as possible according to the law of large numbers. This choice of 1000 embouchures is validated a posteriori given the small error bars in Figure 5. Additional "blackbox" properties of the objective functions under consideration include:

- The evaluation of $J(\mathbf{x})$ may fail due to difficulties to simulate notes (find virtual embouchures).

- It is not possible to reliably compute the gradient of $J(\mathbf{x})$ because of the random selection process in the selection of the virtual embouchure.

- The evaluation of the objective function can be computationally expensive (between 3 and 20 minutes depending on the processor).

- We cannot assume smoothness of the objective (or constraint) functions.

To address these issues, we resort to the use of derivative-free optimization algorithms and a surrogate-assisted modeling strategy.

\section{SURROGATE-ASSISTED DERIVATIVE-FREE OPTIMIZATION}

We use the rigorous derivative-free mesh adaptive direct search (MADS) optimization algorithm, which has convergence properties (Audet and Dennis, Jr., 2006) and has been implemented in the NOMAD software package (Le Digabel, 2011). Every iteration of the MADS algorithm consists of two steps: the optional search and the mandatory poll. The search step can implement any user-defined strategy to obtain promising candidates. The poll step determines candidates around the incumbent solution; it ensures the convergence of the algorithm towards a local optimum. Our strategy in the search step is to formulate and solve a surrogate problem to obtain 
a promising candidate, i.e., we use surrogate models of the computationally-intense simulation procedure to evaluate the objective and constraint function values. We then evaluate the real potential of this promising candidate using the physics-based simulations. In addition, when the MADS algorithm needs to proceed to the poll step, we use the surrogate models to rank-order the poll-generated candidates and then evaluate them opportunistically using the physics-based simulations. In this manner, we generate a large amount of information using computationally inexpensive surrogate models but make algorithmic decisions using the high-fidelity simulations. More details are provided in the next sections.

\section{A. Mesh Adaptive Direct Search}

At each iteration $k$ of the MADS algorithm, the trial points must lie on a mesh $M_{k}$. The mesh size $\Delta_{k}^{m}$ depends on the iteration number $k$ and gets smaller as the optimization converges.

During each search step, a surrogate model $\hat{J}$ is built using previous evaluations of the objective function $J$. Then, a second instance of MADS is used to obtain the design that minimizes $\hat{J}$. This candidate design is then projected on the mesh $M_{k}$ and $J$ is evaluated. If this candidate leads to an improvement of the solution, the surrogate model $\hat{J}$ is updated and the search is repeated. Otherwise, the algorithm continues with the poll step. Two possible surrogate modeling techniques are described in the next section.

During each poll step, a set of candidates $P_{k}$ is generated on the mesh $M_{k}$. The distance between the incumbent solution and the candidates $P_{k}$ is controlled by the poll parameter $\Delta_{k}^{p}$ which, as $\Delta_{k}^{m}$, gets smaller as the optimization converges. The interested reader can refer to (Audet and Dennis, Jr., 2006) for details. As mentioned earlier, we first rank-order the points of the set $P_{k}$ using the 
surrogate model $\hat{J}$. The physics-based sound simulation model $J$ is then used to evaluate the points of the set $P_{k}$ using an opportunistic strategy: If a point is feasible and leads to an improvement of the objective function, the evaluation process is aborted and the algorithm iterates. Note that if a more feasible point is found during the poll step, the mesh and poll parameters are increased so that the algorithm can explore other areas of the design space. Otherwise, these parameters are reduced, which means that the iteration will operate in a closer neighborhood of the design space.

\section{B. Surrogate Modeling Strategy}

We consider two surrogate model approaches in this study:

- An ensemble of surrogate models approach where several surrogates of different types and with different modeling parameters are built and updated while selecting the model that fits the data best at each iteration. We use the same ensemble of surrogates as in (Audet et al., 2018) and (Tournemenne et al., 2017): 6 polynomial regression models, 5 kernel smoothing models, and 6 radial basis function (RBF) models. The best model is chosen according to the order-error with cross-validation (OECV) metric presented in (Audet et al., 2018).

- A LOcally WEighted Scatterplot Smoothing (Talgorn et al., 2018) (LOWESS) surrogate model. This model consists of building a local polynomial regression around the point $\mathbf{x}$ where we wish to predict the objective function. In the construction of this local regression, data points close to $\mathbf{x}$ are given more importance than those further away from $\mathbf{x}$. 


\section{APPLICATION}

We consider three problem with ten design optimization variables: Int, SC Int and SC Dyn. We have also considered these problems with two design variables similarly to (Tournemenne et al., 2017), but we ommit them to keep the paper length reasonable. Since it can be useful to optimization novices, we have made it available online at this link.

The initial bore $\mathbf{x}_{0}$ is close to an existing trumpet bore (whose internal diameter has been measured with different balls of decreasing diameter inserted in the trumpet, and a gauge to measure their position inside the instrument).

The design problems are solved with a budget of 200 function (or blackbox) evaluations. This maximum number of function evaluations is defined empirically by observing the evolution of the objective functions in order to minimize computational cost (i.e., avoid unnecessary evaluations that do not improve the function value significantly, see Figure 4). Moreover, to ensure a reliable quantification of the efficiency of the optimization method, each problem is solved 10 times (with different starting points) for each of the 2 surrogate modeling approaches (using either an ensemble of surrogates according to (Audet et al., 2018) or locally weighted regression models according to (Talgorn et al., 2018)). Given that 3 design problems are studied, there is a total of 60 optimization jobs ( 3 problems $\times 2$ surrogate approaches $\times 10$ starting points).

In order to undertake this considerable computational endeavor, we relied on a high performance computing cluster, parallelizing the sound simulations on 2 Haswell Intel $₫$ Xeon $₫$ E5$2680 \mathrm{v} 32.5 \mathrm{GHz}$ processors providing 24 cores for each of the 60 jobs. One evaluation of the objective function took between $2 \mathrm{~min} 40 \mathrm{sec}$ and $3 \mathrm{~min} 50 \mathrm{sec}$ for Int and SC Int (4000 sounds 
simulated), depending on the difficulty to simulate sounds for the considered geometry $\mathbf{x}$. We provide sounds of the studied notes of the initial trumpet as supplementary materials ${ }^{1}$. These sounds are made of the first 6 harmonics of the permanent regime (no transients).

\section{A. Design Optimization Problem with 10 Variables (10d problem)}

The ten design variables represent the geometry of the leadpipe which is an important part of the bore that connects the mouthpiece to the tuning slide. The leadpipe, roughly conical, has a significant influence on the intonation and timbre of the instrument (Petiot and Gilbert, 2013). Eleven parts of equal length $(l=20 \mathrm{~mm})$ are considered. The design variables are the inner radii of the leadpipe at the connection between two parts (10 variables out of 12 control points because the initial and last control points are fixed at 4.64 and $5.83 \mathrm{~mm}$, respectively). These 10 inner radii values span from 4.5 to $6 \mathrm{~mm}$. The rest of the instrument corresponds approximately to a standard trumpet.

In this realistic design space, the high dimensionality requires efficient optimization: a discretization of the space with a granularity of $0.5 \mathrm{~mm}$ ( $20 \%$ of the range of each dimension) would necessitate $4^{10}$ function evaluations (more than 1 million, which is not tractable in a reasonable computation time).

Figure 4 presents the performance of the optimization approaches for the three 10 -d problem formulations. The initial value for $J_{1}$ is 8.7 cents. The initial value for $J_{2}$ and $J_{3}$ are 2.23 and 0.04 (value of SC), respectively, as can be seen in Figure 5.

On average, the LOWESS surrogate approach yields slightly better designs for $J_{1}(\mathbf{x}, \varphi)$ and better results for $J_{3}(\mathbf{x}, \varphi)$. For Int $\left(J_{1}\right)$ the best objective function value obtained is 0.1 cents, 

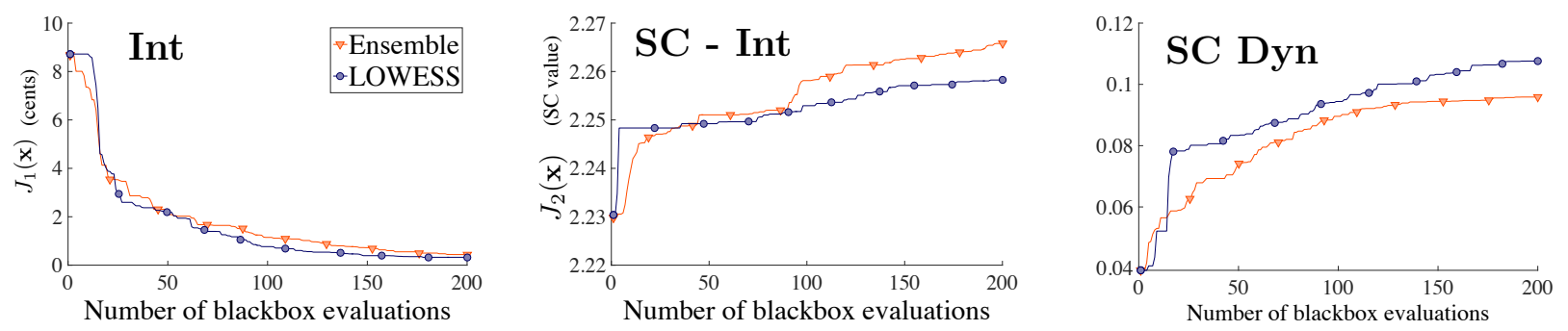

FIG. 4. Evolution of the objective function values for the $310-\mathrm{d}$ problem formulations; from left to right:

Int, SC Int, and SC Dyn (color online)

and the best intonation improvement is 8.6 cents. This represents a $99 \%$ improvement. For SC Int $\left(J_{2}\right)$ the best objective function value obtained is 2.27 (value of SC), and the average spectral centroid improvement is 0.04 . This represents a $1.8 \%$ overall improvement $((2.27-2.23) / 2.23))$. For SC Dyn $\left(J_{3}\right)$ the best objective function value obtained is 0.14 (value of SC), and the spectral centroid difference improvement is 0.1 . This optimal bore improves 35 times the $J_{3}$ performance of the initial bore $(0.14 / 0.04)$. Yet, measurements on an expert trumpet player playing $\mathrm{B} b 4$ concert pitch show a SC increase around 0.8 between a $p$ and $f f$ note for the 6 first harmonics, which is more than 5 times superior to the simulated values of the optimum. These results are in accordance with (Petiot and Gilbert, 2013). Even if the method optimizes the spectral centroid variation, the model does not allow the prediction of realistic spectral centroid values. Non-linear propagation in the bore should be taken into account.

Figure 5 provides a finer acoustical analysis of these results showing the contributions of each note to the objective functions for the initial bore and the optimal bore. The error bars are computed following the guidelines of the Joint Committee for Guides in Metrology (JCGM, 2008). For Int, the $\mathrm{Bb} 3$ and F4 show significant improvements of 7.5 and 13.6 cent, respectively, which are supe- 

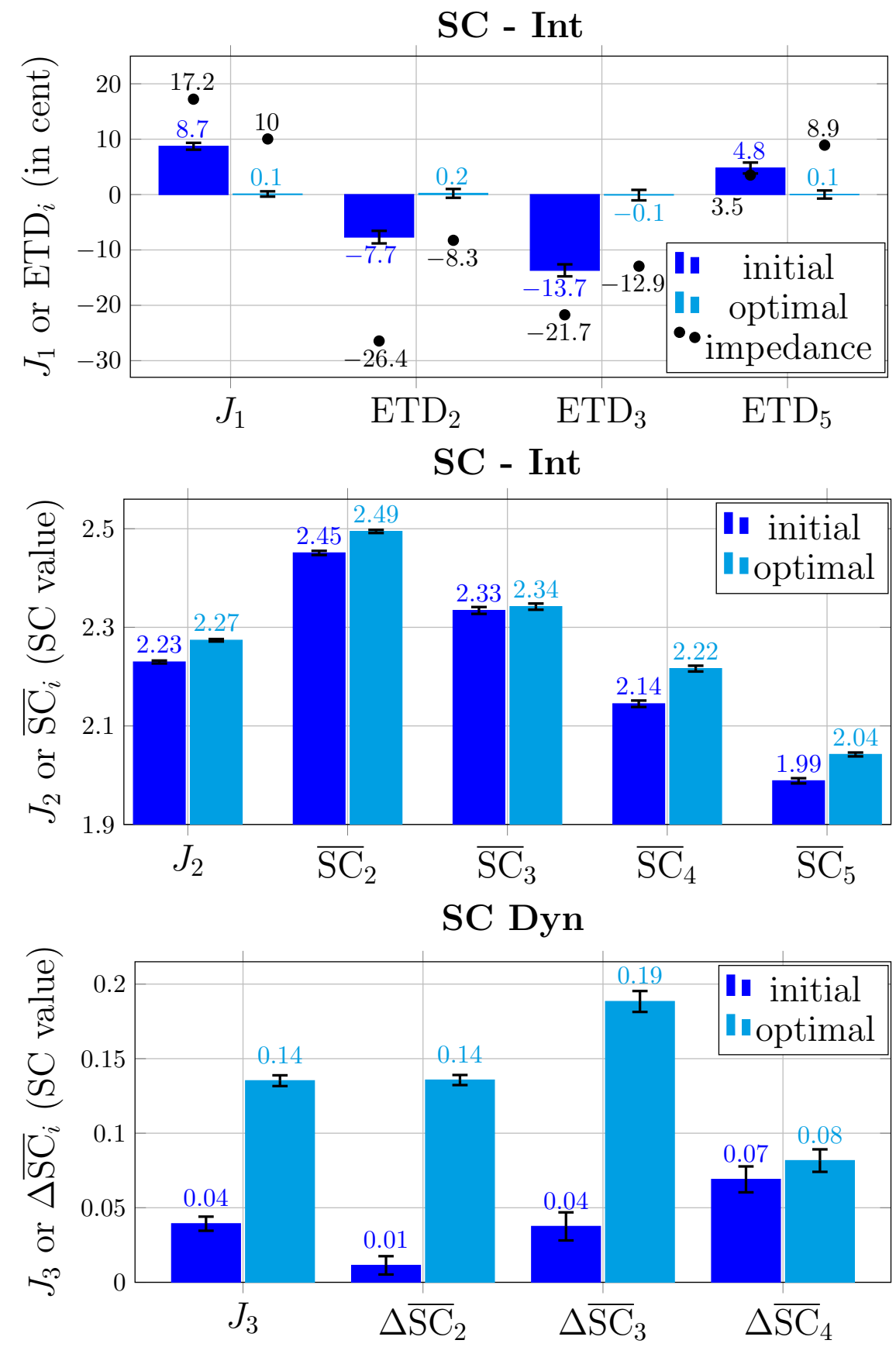

FIG. 5. On the top part, the columns represent from left to right $J_{1}$ value and the non zero ETDs. Two bores are evaluated, the initial bore of all the optimization jobs and the best bore from the most successful job (out of the 20 available). The error bars equal to 2 standard deviations of the estimated quantity. Equivalent plots for the design problems SC - Int and SC Dyn are drawn below. $\overline{\mathrm{SC}}_{i}$ is $\overline{\mathrm{SC}}$ restricted to $\varphi_{i}$. In addition, the values of $J_{1}$ and the corresponding ETDs, obtained with the input impedance peaks frequency values, are presented in black dots. 
rior to the classical just-noticeable difference (JND) of 5 cents. $\mathrm{Bb} 4$ being the relative reference, it is always considered perfectly in tune. Since the initial value of D5 is already low (4.8 cent) the improvement of 4.7 cent is slightly lower than the JND. The sound simulation results concerning intonation are in agreement with the impedance peaks frequency values except for D5, for which the optimal trumpet seems less in tune when considering the impedance peaks frequency values. For SC - Int, the highest improvement is 0.08 (value of SC) for Bb4 which is at the same level than the JND of 0.1 reported in (Jeong and Fricke, 1998). Consequently, for Bb3, F4 and D5, the improvements seem negligible, rising to 0.04, 0.01 (error bars level), and 0.5, respectively. For SC Dyn, the improvements for Bb3 and F4 are above the JND ( 0.13 and 0.15 , respectively), contrary to $\mathrm{Bb} 4(0.01)$.

Figure 6 shows the leadpipes yielding the best value for each design problem.

As in (Tournemenne et al., 2017), the optima are counter intuitive since the leadpipe does not have a positive slope along the whole trumpet axis. This kind of shape for a leadpipe is not common among trumpets because it is very difficult to manufacture. The optimization algorithm was able to explore the design space in order to find unusual designs. Finally, the geometrical differences between the 3 optima and the initial leadpipe are on the order of the millimeter for several control radii, which will lead to noticeably different instruments when manufactured.

\section{B. Discussion}

Concerning intonation, the objective function value based on sound simulations is always below the one obtained only with the input impedance peaks (cf. black dots of Figure 5). This has been verified on more examples (Tournemenne, 2017), and would mean that the musician always plays 


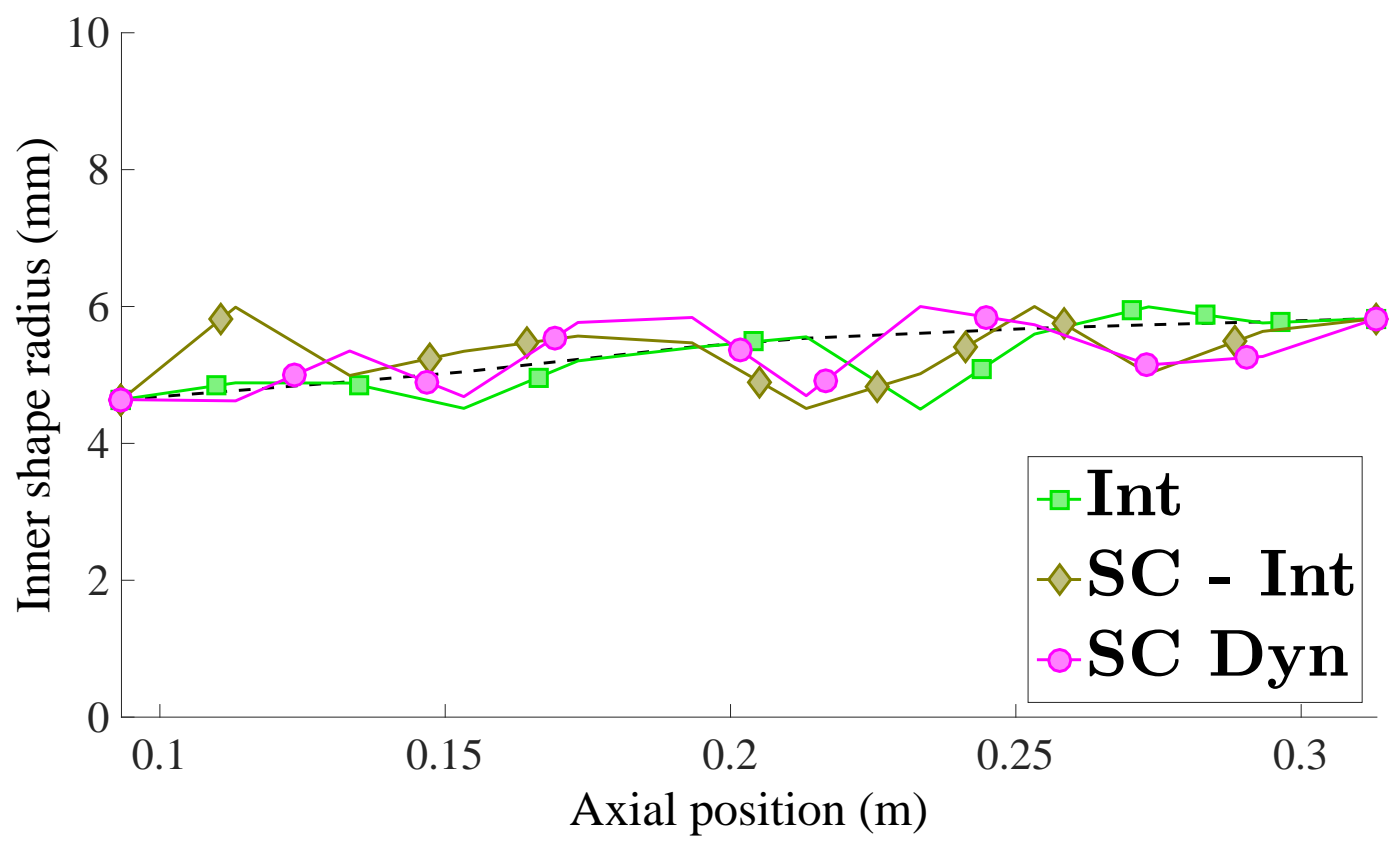

FIG. 6. Representation of the leadpipe inner radius along the instrument axis; the black dotted line to the initial geometry (measured on our trumpet); each other line corresponds to the best design found by each objective over 20 jobs (color online)

more in tune that would suggest the resonance frequency values of the input impedance. This is necessarily an effect of the non-linear part of the model which requires further study. The history plot of the Int design problem demonstrates a significant intonation improvement, above JND: the objective function is decreased by $99 \%$ to 0.1 cents. Such a level of performance has been achieved for a small price (200 evaluations compared with the million evaluations needed by brute force). Future studies may consider even more design variables. Concerning the spectral centroid, the results show improvements of the objective function $J_{2}$ and $J_{3}$, even if they remain limited and close to the JND. More marked improvements would certainly occur on real trumpets when played. 
Regarding the surrogate modeling approach, it is hard to tell whether one is consistently better than the other as they seem to exhibit the same exploration to exploitation ratio of the design space. It should be noted that the locally weighted regression models take longer to compute than the ensemble of surrogates in high-dimensional problems (in our case, the difference is one hour vs. few minutes for ten variables).

\section{CONCLUSIONS}

In this paper, we extended a new paradigm for design optimization of brass instruments. This new paradigm goes beyond impedance based optimization.(Braden et al., 2009; Guilloteau, 2015; Kausel, 2001; Macaluso and Dalmont, 2011; Noreland et al., 2013, 2010; Poirson et al., 2007). We consider the optimization of objective functions (possibly subject to constraints) based directly on the sounds spectrum, which, to the best of our knowledge, is a novel approach. The originality of the approach lies in the fact that the objective function is not limited to a characterization of the instrument alone, but includes virtual musicians in an physical model, to optimize directly the instrument sounds. The main contribution of this paper is to demonstrate how physics-based sound simulations can be integrated in an iterative optimization algorithm, which requires that simulations converge automatically toward auto-oscillations for every considered bore of the design space, without any assistance of the user. A second contribution concerns the optimization of objective functions based on a particular dimension of the timbre of the sounds, the spectral centroid. Applied to the optimization of a trumpet, the results show that the optimization method, based on the MADS algorithm, is efficient to define optimal solutions in a reasonable computation time, with or without constraints, for problems up to 10 design variables. 
While the approach shows promising potential, there is room for improvement. Even if it is efficient to reproduce differences between instruments concerning intonation and spectral centroid, the elementary model could be improved to generate a more realistic sound spectrum. The sound optimized in this work is the sound in the mouthpiece. Even if this does not change the principle of the method presented, it could be interesting to define a relevant radiated pressure outside the instrument, and to include it in the optimization considerations. Another valuable contribution to this numerical study concerns the manufacturing of the optimal instruments and their objective and subjective study. This would help evaluate actual improvement. Regarding implementation, the influence of the selection process of the virtual embouchure could be investigated further, as it may provide more robust descriptors of the ease of playing of the considered instruments. Regarding the methodology, a study of temporal sound simulations could lead to new classes of objective functions, such as attack times. More ambitious still, the inclusion of non-linear propagation in simulations would produce more realistic sounds improving the objective functions considered in this work. Finally, the main challenge in the design of musical instrument lies in the definition of judicious objective functions providing actual insight of the instrument intrinsic quality. This task may be accomplished by working side-by-side with instrument makers whose empirical understanding may be translated to computational principles and models.

\section{ACKNOWLEDGMENTS}

The authors are grateful to the NOMAD team (Prof. Charles Audet, Prof. Sébastien Le Digabel and Dr. Christophe Tribes), Prof. Saïd Moussaoui for his help with the optimization problem formulation, Prof. Jean-Pierre Dalmont for his precious help with the input impedance models, 
and Juliette Chabassier for the many helpful discussions. Numerical experiments were carried out using the PlaFRIM experimental testbed (see https://www.plafrim.fr/).

${ }^{1}$ See Supplementary materials at [URL will be inserted by AIP] for the sounds.

Adachi, S., and Sato, M.-a. (1996). “Trumpet sound simulation using a two-dimensional lip vibration model," The Journal of the Acoustical Society of America 99(2), 1200-1209.

Audet, C., and Dennis, Jr., J. E. (2006). "Mesh adaptive direct search algorithms for constrained optimization,” SIAM Journal on Optimization 17(1), 188-217.

Audet, C., Kokkolaras, M., Le Digabel, S., and Talgorn, B. (2018). “Order-based error for managing ensembles of surrogates in derivative-free optimization," Journal of Global Optimization 70(3), 645-675.

Benade, A. H. (1966). "Relation of AirColumn resonances to sound spectra produced by wind instruments," J. Acoust. Soc. Am. 40(1), 247-249.

Boutin, H., Fletcher, N., Smith, J., and Wolfe, J. (2015). "Relationships between pressure, flow, lip motion, and upstream and downstream impedances for the trombone," The Journal of the Acoustical Society of America 137(3), 1195-1209.

Braden, A. C. P., Newton, M. J., and Campbell, D. M. (2009). “Trombone bore optimization based on input impedance targets," J. Acoust. Soc. Am. 125(4), 2404-2412.

Bromage, S., Campbell, M., and Gilbert, J. (2010). "Open areas of vibrating lips in trombone playing," Acta Acustica united with Acustica 96(4), 603-613. 
Campbell, M. (2004). "Brass instruments as we know them today," Acta Acustica united with Acustica 90(4), 600-610.

Caussé, R., Kergomard, J., and Lurton, X. (1984). "Input impedance of brass musical instruments_comparison between experiment and numerical models," J. Acoust. Soc. Am. 75(1), 241-254.

Chen, F.-C., and Weinreich, G. (1996). "Nature of the lip reed," The Journal of the Acoustical Society of America 99(2), 1227-1233.

Cullen, J., Gilbert, J., and Campbell, M. (2000). "Brass instruments: Linear stability analysis and experiments with an artificial mouth,” Acta Acustica united with Acustica 86(4), 704-724.

Deutsch, D. (2013). The Psychology of Music (Third Edition) (Academic Press).

Elliott, S., and Bowsher, J. (1982). "Regeneration in brass wind instruments," Journal of Sound and Vibration 83(2), 181-217.

Eveno, P., Petiot, J.-F., Gilbert, J., Kieffer, B., and Caussé, R. (2014). “The relationship between bore resonance frequencies and playing frequencies in trumpets," Acta Acustica united with Acustica 100(2), 362-374.

Fletcher, N. H., and Tarnopolsky, A. (1999). "Blowing pressure, power, and spectrum in trumpet playing," J. Acoust. Soc. Am. 105(2), 874-881.

Gilbert, J., Kergomard, J., and Ngoya, E. (1989). "Calculation of the steady-state oscillations of a clarinet using the harmonic balance technique," J. Acoust. Soc. Am. 86(1), 35-41.

Guilloteau, A. (2015). "Conception d'une clarinette logique" ("Design of a logical clarinet"), Ph.D. thesis, Aix-Marseille. 
Jeong, D., and Fricke, F. R. (1998). "The dependence of timbre perception on the acoustics of the listening environment," in Proc. 16th Int. Congress on Acoustics and 135th Meeting of the Acoustical Society of America, Vol. 3, pp. 2225-2226.

JCGM (2008). "Evaluation of measurement data - guide to the expression of uncertainty in measurement," Technical Report .

Kausel, W. (2001). "Optimization of brasswind instruments and its application in bore reconstruction," Journal of New Music Research 30(1), 69-82.

Le Digabel, S. (2011). "Algorithm 909: NOMAD: Nonlinear optimization with the MADS algorithm,” ACM Trans. Math. Softw. 37(4), 44:1-44:15.

Macaluso, C. A., and Dalmont, J.-P. (2011). "Trumpet with near-perfect harmonicity: design and acoustic results,” J. Acoust. Soc. Am. 129(1), 404-414.

Martin, D. W. (1942). "Directivity and the acoustic spectra of brass wind instruments," The Journal of the Acoustical Society of America 13(3), 309-313.

Myers, A., Pyle Jr, R. W., Gilbert, J., Campbell, D. M., Chick, J. P., and Logie, S. (2012). "Effects of nonlinear sound propagation on the characteristic timbres of brass instruments," The Journal of the Acoustical Society of America 131(1), 678-688.

Newton, M. J., Campbell, M., and Gilbert, J. (2008). "Mechanical response measurements of real and artificial brass players lips," J. Acoust. Soc. Am. 123(1), EL14-20.

Noreland, D., Kergomard, J., Laloë, F., Vergez, C., Guillemain, P., and Guilloteau, A. (2013). "The logical clarinet: Numerical optimization of the geometry of woodwind instruments," Acta Acustica united with Acustica 99(4), 615-628. 
Noreland, J. O. D., Udawalpola, M. R., and Berggren, O. M. (2010). “A hybrid scheme for bore design optimization of a brass instrument,” J. Acoust. Soc. Am. 128(3), 1391-1400.

Petiot, J.-F., and Gilbert, J. (2013). “Comparison of trumpets' sounds played by a musician or simulated by physical modelling," Acta Acustica united with Acustica 98, 475-486.

Poirson, E., Petiot, J.-F., and Gilbert, J. (2005). "Study of the brightness of trumpet tones," The Journal of the Acoustical Society of America 118(4), 2656-2666.

Poirson, E., Petiot, J.-F., and Gilbert, J. (2007). "Integration of user perceptions in the design process: Application to musical instrument optimization," Journal of Mechanical Design 129(12), $1206-1214$.

Talgorn, B., Audet, C., Le Digabel, S., and Kokkolaras, M. (2018). “Locally weighted regression models for surrogate-assisted design optimization," Optimization and Engineering 19(1), 213238.

Tournemenne, R. (2017). "Optimisation d'un instrument de musique de type cuivre basée sur des simulations sonores par modèle physique" ("Brass instrument optimization based on physicsbased sound simulations”), Ph.D. thesis, École Centrale de Nantes.

Tournemenne, R., Petiot, J.-F., Talgorn, B., Kokkolaras, M., and Gilbert, J. (2017). “Brass instruments design using physics-based sound simulation models and surrogate-assisted derivativefree optimization,” Journal of Mechanical Design 139(4), 041401.

Velut, L., Vergez, C., Gilbert, J., and Djahanbani, M. (2017). "How well can linear stability analysis predict the behaviour of an Outward-Striking valve brass instrument model?," Acta Acustica united with Acustica 103(1), 132-148. 
Brass Instrument optimization

${ }_{572}$ Yoshikawa, S. (1995). “Acoustical behavior of brass player's lips," the Journal of the Acoustical 573 Society of America 97(3), 1929-1939. 